Supporting Information

\title{
Micelle-laden hydrogel microparticles for the removal of hydrophobic micropollutants from water
}

Devashish Gokhale ${ }^{1}$ Ian Chen ${ }^{2}$, and Patrick S. Doyle ${ }^{1 *}$

${ }^{1}$ Department of Chemical Engineering, Massachusetts Institute of Technology, Cambridge, MA 02139, USA

${ }^{2}$ Department of Materials Science and Engineering, Massachusetts Institute of Technology, Cambridge, MA 02139, USA

*Email: pdoyle@mit.edu
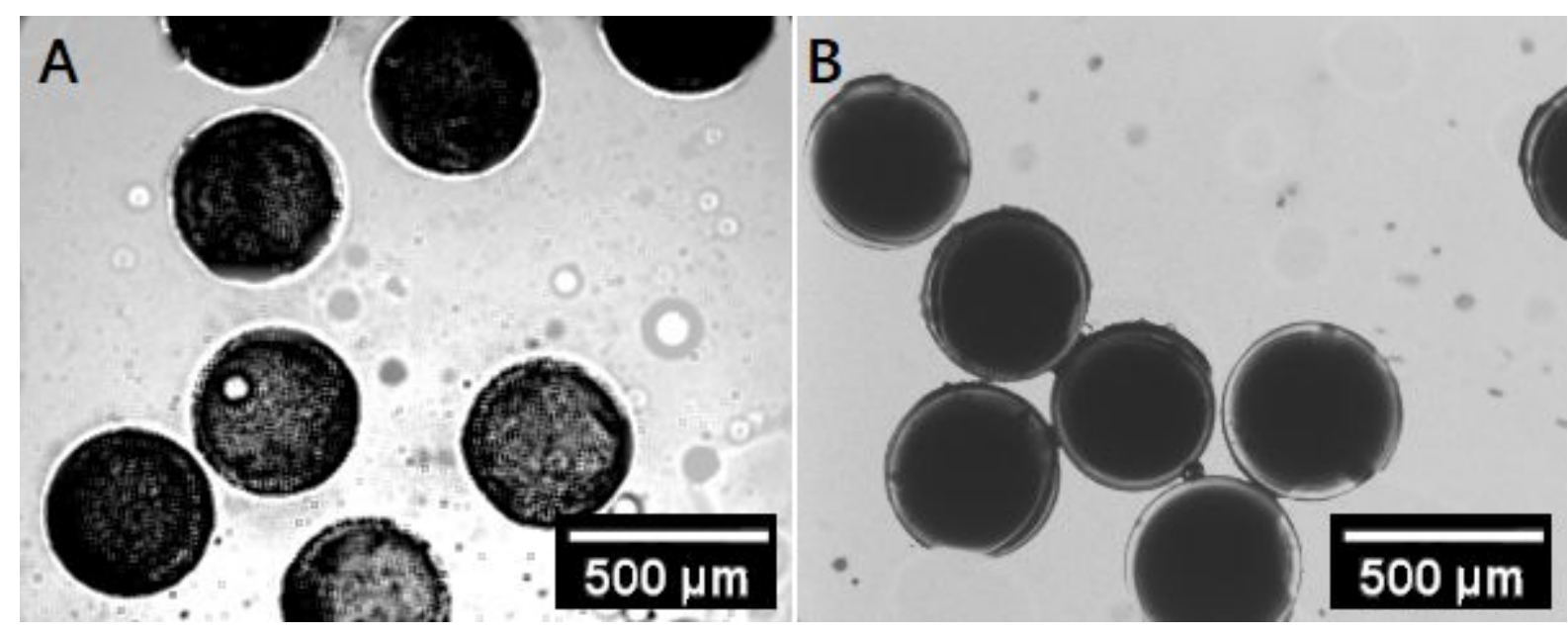

Figure S1: 10\% PEGDA, 5\% B25MA, 5\% PI microparticles (A) 1 hour and (B) 1 year after synthesis. 


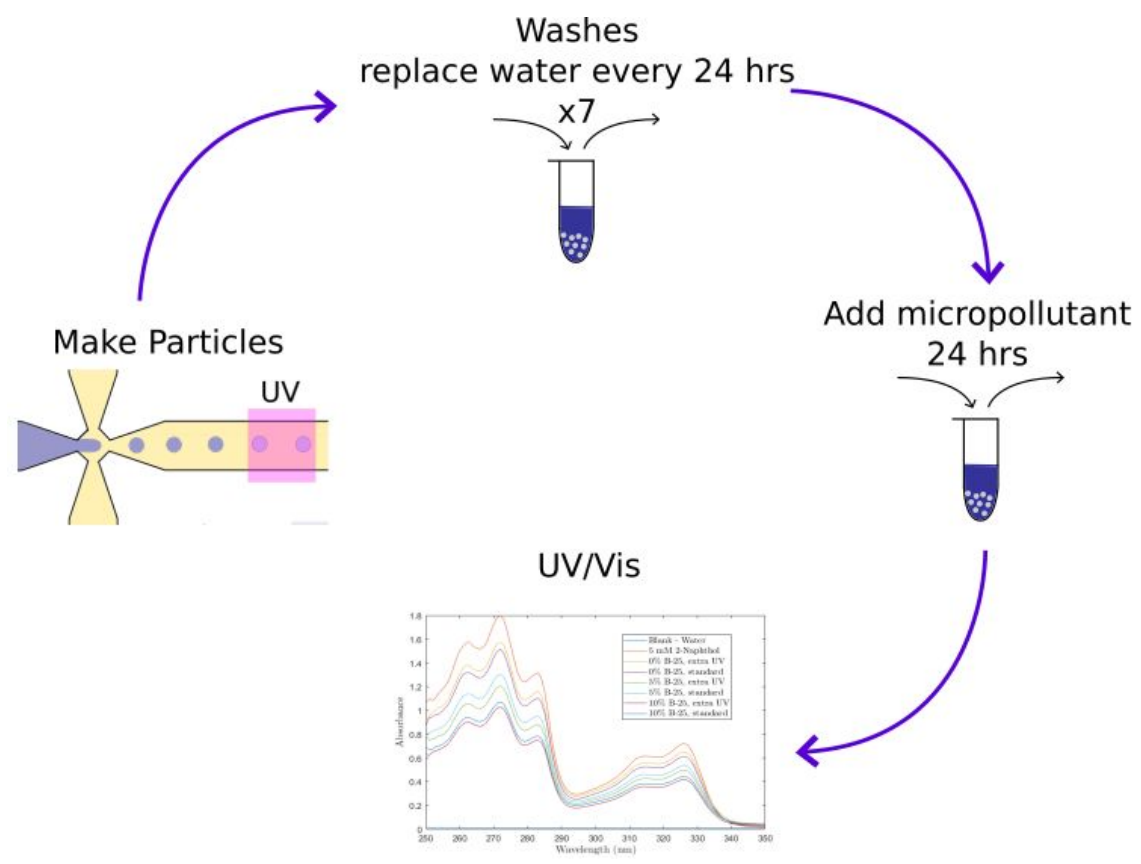

Figure S2: Equilibrium uptake experiment schematic.
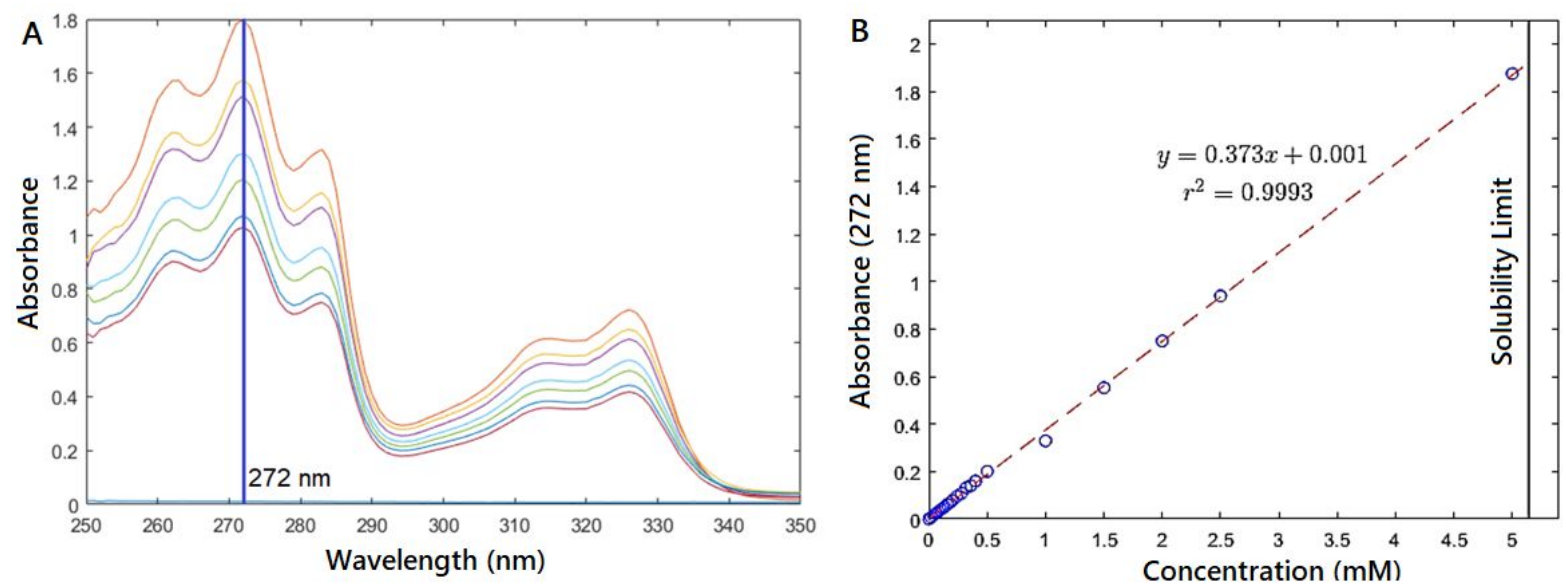

Figure S3: 2-Naphthol absorbance spectra (A) and a Beer-Lambert calibration curve based on the peak at $272 \mathrm{~nm}$. Other peaks, like the one at $326 \mathrm{~nm}$, may also be used to prepare calibrations. 


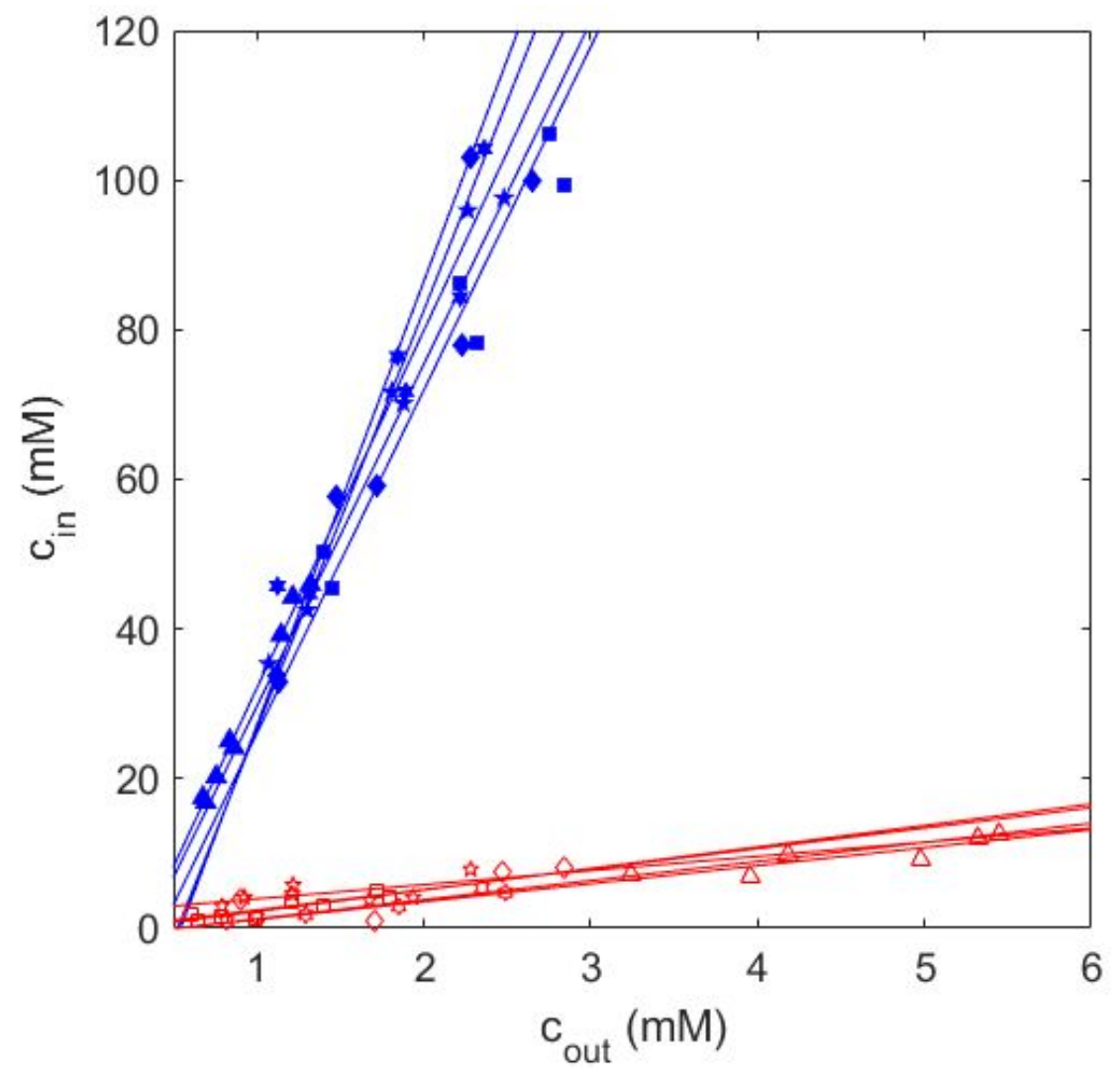

Figure S4: Effect of using a water-ethanol mixture as the solvent in the F127DA monomer solution. All plots are for 10\% PEGDA, 5\% PI and 15\% F127DA in water-ethanol mixtures of varying ethanol fraction, indicated by various symbols. Equilibrium isotherms are shown with water (blue, filled) and ethanol (red, unfilled) as the supernatants. The presence of ethanol does not significantly affect the equilibrium isotherms. 

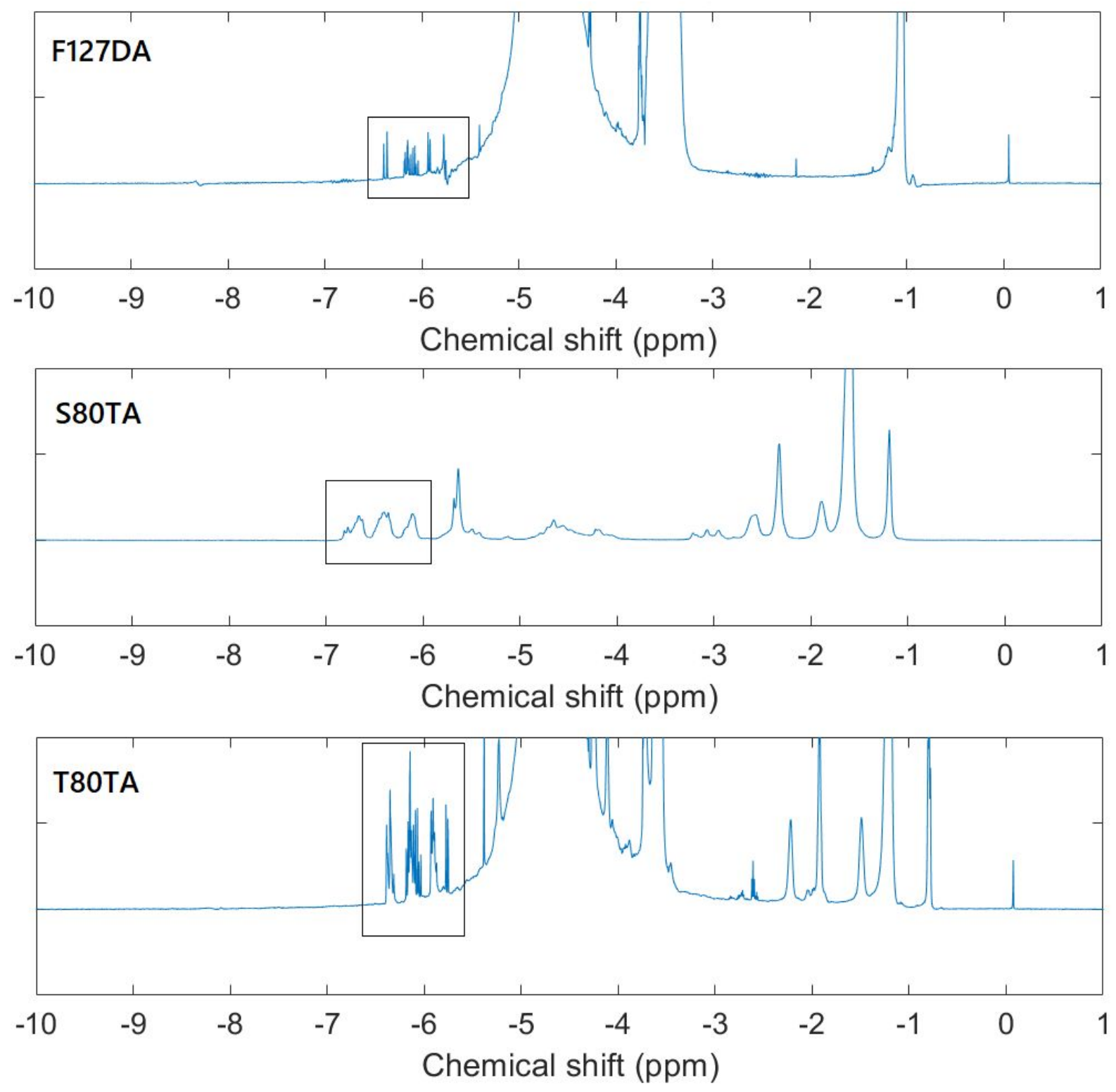

Figure S5: NMR spectra for F127DA, S80TA, and T80TA after acrylation. Note the appearance of acrylate peaks (black boxes), which are absent in the NMR spectra of the unacrylated surfactants. The areas under the peaks can be used to quantify the number of acrylate groups added relative to the number of surfactant molecules, and hence evaluate conversion. 


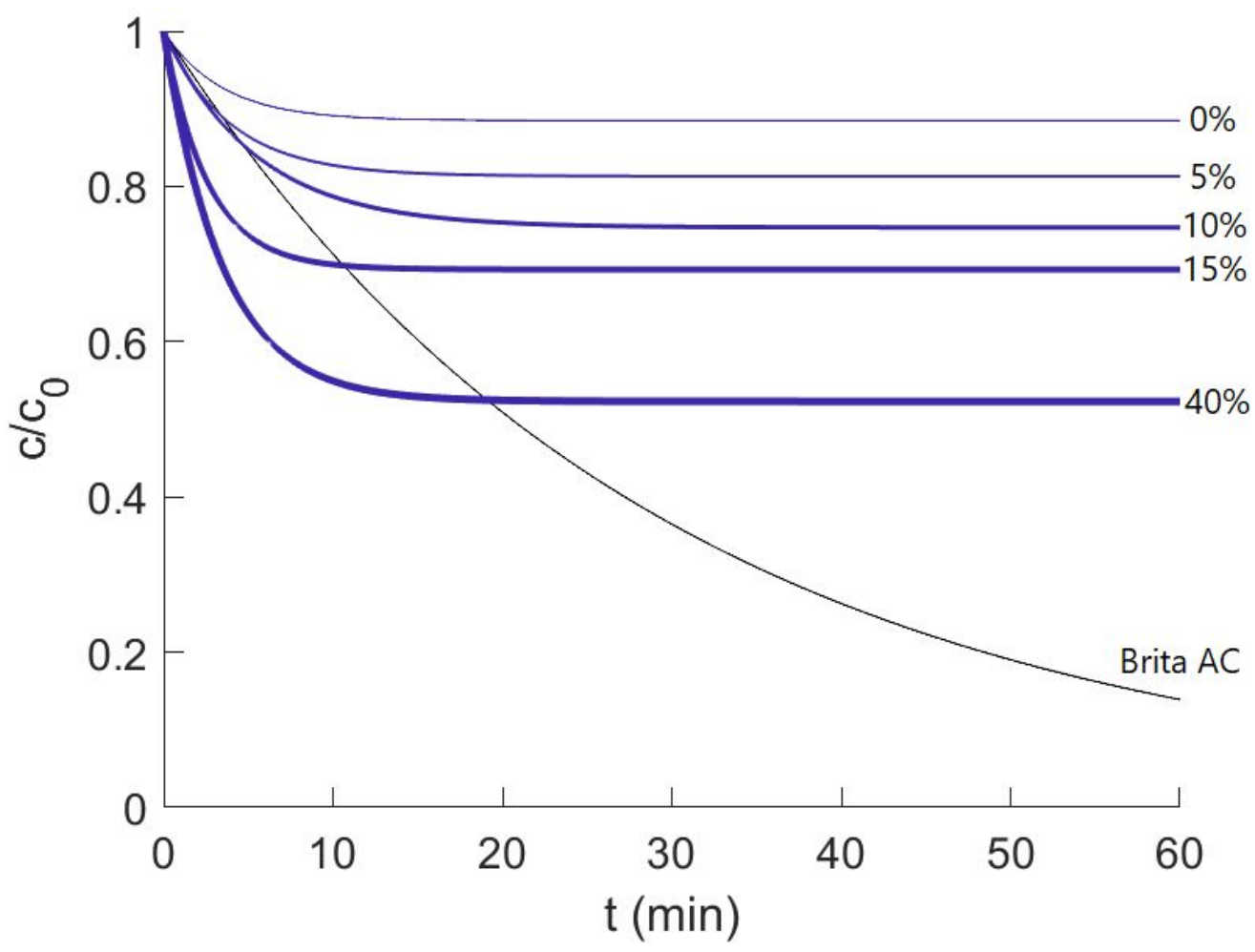

Figure S6: Kinetics of micropollutant (2-naphthol) uptake by hydrogel microparticles containing varying amounts of F127DA micelles, compared to commercial activated carbon (Brita AC) at long times. 


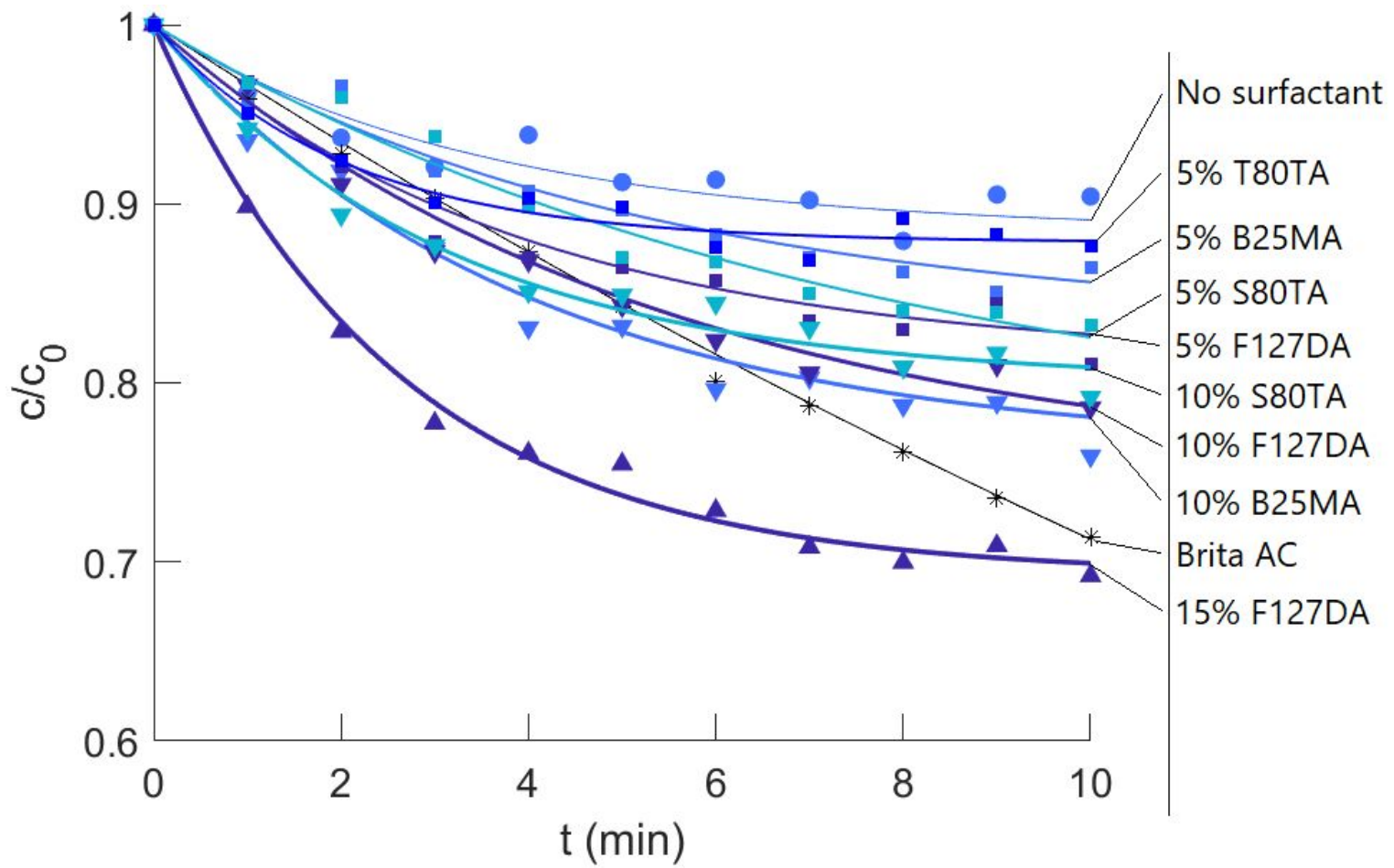

Figure S7: Kinetics of micropollutant (2-naphthol) removal - all data sets in figure 4 combined for unified viewing

Critical micelle concentrations of common surfactants of interest

\begin{tabular}{|c|c|c|}
\hline \multicolumn{2}{|c|}{ Surfactant } & CMC (mol/L) \\
\hline \multicolumn{3}{|c|}{ Alkyl-PEO surfactants } \\
\hline B25 & NEO-PPO-PEO surfactants \\
\hline & & $2.80 \mathrm{E}-06$ \\
\hline F127 & Sorbitan-ester surfactants \\
\hline Span 80 & & $2.80 \mathrm{E}-04$ \\
\hline Tween 80 & & $9.90 \mathrm{E}-06$ \\
\hline
\end{tabular}




\section{Derivation of uptake kinetics equation}

Let $c$ be the pollutant concentration in solution at time $t$, with initial value $c_{0} . K$ is the partition coefficient, $k_{c} a$ is the mass transfer coefficient, and $c_{s}$ is the concentration at time $t$ at the particle-liquid interface. Let the average concentration inside the particles be $q . V$ is the volume of the supernatant and $V_{p}$ is the volume of the particles. Then, we have the following equations based on equilibrium, mass transfer and pollutant mass balance respectively.

$$
\begin{gathered}
q=K c_{s} \\
\frac{d c}{d t}=k_{c} a\left(c-c_{s}\right) \\
q=\frac{V}{V_{p}}\left(c_{0}-c\right)
\end{gathered}
$$

Combining these, we get

$$
\frac{d c}{d t}=k_{c} a\left(\left(1+\frac{V}{V_{p} K}\right) c-\frac{V}{V_{p} K} c_{0}\right)
$$

Solving this differential equation for $c(t)$, with initial condition $c(0)=c_{0}$ yields

$$
\frac{c(t)}{c_{0}}=\frac{\frac{V}{V_{p} K}+e^{-\left(1+\frac{V}{V_{p} K}\right) k_{c} a t}}{\frac{V}{V_{p} K}+1}
$$

